wrong (spin-non-conserving) path, the ion is able to emit on average 700 photons while keeping its electron spin state.

Finally, the two spin-conserving optical transitions have different optical wavelengths and can be addressed separately by a narrow-band single-mode laser. If, say, the electron is in the ground state $|\uparrow\rangle$ and one shines the laser in resonance with the $|\uparrow\rangle-|\uparrow\rangle$ transition, the erbium ion keeps emitting fluorescence photons until it accidentally jumps into the $|\downarrow\rangle$ state. By observing this fluorescence, one can immediately say that the spin is in state $|\uparrow\rangle$. However, if there is no fluorescence emitted, one concludes that the spin is in state $|\downarrow\rangle$. This simple but elegant strategy allows the observation of quantum jumps of erbium electron spin in real time - with very far reaching implications for quantum computing architectures based on REIs.

While both groups of authors have used similar methods for cavity-enhanced emission, and have measured spin relaxation using the coupled spin-cavity system, Kindem and colleagues went one step beyond - measuring spin dephasing and even exploring the spin dephasing limits in YVO with spin decoupling techniques.

Besides the intriguing physics and engineering achievements behind the observation of spin quantum jumps, the ability to read out the quantum state of the spin is of fundamental importance for quantum information processing. Certain algorithms that are critical for scalability, such as error correction, rely on singleshot readout. While the detection and coherent control of REI has been elusive for a long time, with the invention of efficient resonators for REI-based systems published now, REIs are quickly catching up with the leading contenders in the field. Starting from the first detection of single REI reported eight years ago ${ }^{5}$, most basic qubit functionalities have now been shown, although some key steps such as spin-photon and entanglement of remote REIs remain out of reach for now. However, owing to the diversity of REI species, the possibility of their tight packing into the crystal and the ultra-long coherence times, the future of REI-based quantum hardware looks bright.

Roman Kolesov and Jörg Wrachtrup (D) 3. Physikalisches Institut, Universität Stuttgart, Stuttgart, Germany.

e-mail: roman.kolesov@pi3.uni-stuttgart.de; joerg.wrachtrup@pi3.uni-stuttgart.de

Published online: 30 March 2020 https://doi.org/10.1038/s41567-020-0871-3

References

1. Afzelius, M., Gisin, N. \& de Riedmatten, H. Phys. Today 68, 42 (2015).

2. Zhong, M. et al. Nature 517, 177-180 (2015).

3. Raha, M. et al. Nat. Commun. https://doi.org/10.1038/s41467020-15138-7 (2020).

4. Kindem, J. M. Nature https://doi.org/10.1038/s41586-020-2160-9 (2020).

5. Kolesov, R. et al. Nat. Commun. 3, 1029 (2012).

\title{
Mode-locking dissected
}

\author{
Despite the wide use of mode-locked lasers, no general theory for mode-locking exists. An attractor dissection \\ approach provides some intuitive understanding of the complex dynamics in one type of mode-locking.
}

\section{F. Ömer Ilday}

M ode-locked lasers have extraordinarily diverse scientific, medical and industrial applications, and have directly enabled three Nobel Prizes. Until recently, mode-locking was restricted to longitudinal modes only and required laser cavities with a single transverse mode. But simultaneous locking of longitudinal and spatial modes of a laser - spatiotemporal mode-locking - is also possible. Writing in Nature Physics ${ }^{1}$, Logan Wright and co-workers have now taken a first and bold step towards a theory of spatiotemporal mode-locking.

A laser cavity (Fig. 1a) generally supports many distinct electromagnetic modes, both in the longitudinal and transverse directions. Most commonly, these modes oscillate independently of each other. The laser's output can be nearly constant in time, but with large fluctuations, and the ability to focus its beam is limited. From the humble laser pointer to ultra-high-power lasers for cutting metal sheets, multimode lasers are widely used. The straightforward - although technically challenging alternative is to restrict the cavity to a single transverse and, less commonly, a single longitudinal mode. A single-mode laser can produce a truly constant output, and its beam can be focused to the diffraction limit. These lasers are the workhorses of many scientific applications, including gravitational wave detection and lasercooling of atoms.

The not-so-straightforward but fascinating alternative is mode-locking. Under the right conditions, thousands of longitudinal modes of a cavity can spontaneously self-organize to oscillate in perfect synchrony with each other, like an enormous orchestra. The synchronized oscillation of the modes enables their coherent superposition to generate ultrashort pulses, which have found a diverse range of scientific and industrial applications. The recent demonstration of spatiotemporal mode-locking - the simultaneous locking of longitudinal and transverse modes of a laser ${ }^{2}$ - came as a surprise. This advance has created much excitement in the laser community; thanks to the increased dimensionality, we are likely to witness an explosion in the richness of self-organization that lasers can exhibit.

However, this richness is also a challenge - without theoretical guidance, exotic mode-locking states may turn into the proverbial needle in a haystack. For example, one trustworthy tool to build intuition about mode-locking is numerical simulation of the laser's operation. In spatiotemporal 


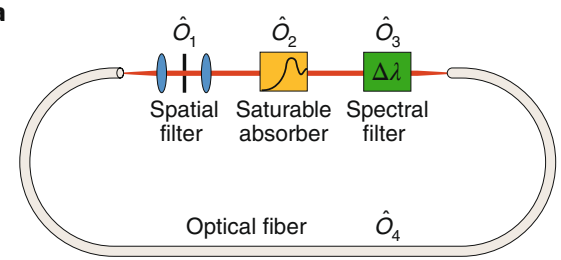

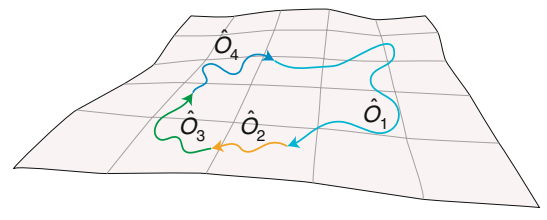

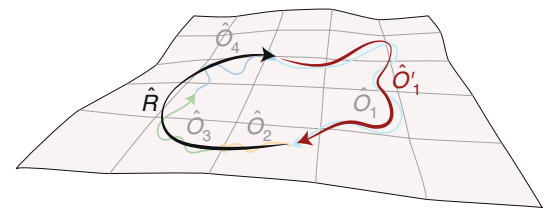

Fig. 1 | Spatiotemporal mode-locking in phase space. a, A mode-locked laser's cavity contains multiple optical elements, each described by a nonlinear operator $\left(\hat{O}_{1}, \hat{O}_{2}, \hat{O}_{3}, \hat{O}_{4}\right)$. b. Evolution of the pulse through the laser cavity constitutes a closed trajectory in phase space, described by the operators $\hat{O}_{1}, \hat{O}_{2}, \hat{O}_{3}$ and $\hat{O}_{4} \cdot \mathbf{c}$, An attractor dissection approach produces a highly simplified model by retaining one or two dominant operators $\left(\hat{O}_{1}{ }^{\prime}\right)$ and replacing the others with a simple rescaling operation $(\hat{R})$.

mode-locking, one has to solve as many coupled equations as there are spatial modes. Each of these equations is as rich and complicated as the single one used to model regular mode-locking - which is itself still not fully understood - not to mention the superlinear increase of the number of parameters with the number of modes.

Even regular mode-locking does not have a general formulation, so the task Wright and co-workers have accomplished may look impossibly difficult. But sometimes a more general problem can yield more insight than its more restricted version. Indeed, their method - which they call attractor dissection - allows them to break down overwhelmingly complicated dynamics into simpler scenarios.

A laser evolves towards the state that minimizes its losses ${ }^{1,3}$. Various distinct effects are acting on the pulse as it traverses the cavity (Fig. 1b). A suitable nonlinear operator can describe each effect. But, if all of them are considered at once, figuring out which final state minimizes the losses is often impossible. The attractor dissection method simplifies finding this state. Crucially, Wright and co-workers intuited that the ultimate selection of a specific mode-locked state by the lowestloss principle is often, but not always, dominated by one or two of these effects. For the parameter ranges where this is true, they focused on the dominant operator and lumped together the remaining effects into a simple rescaling operator (Fig. 1c). This highly simplified model can be analysed and understood intuitively. For example, the spatial filter alone may be dominant for a given set of cavity parameters. For another set, the saturable absorber together with the gain may dominate. By playing around with various possible scenarios, the team was able to predict previously unknown regimes of spatiotemporal mode-locking, which they subsequently demonstrated experimentally.

This theory is not the final word on spatiotemporal mode-locking. The occasionally non-rigorous but beautifully insightful attractor dissection approach will surely stimulate new work. Besides several more specialized questions, one fundamental question remains unanswered: the well-known dictum that a laser evolves towards lowest loss generally holds, but even for regular mode-locking there is no rigorous proof. It is far from clear how well it holds in complex situations like spatiotemporal mode-locking, with higher-dimensional phase space. For example, the laser can have access to multiple steady states that extract gain with sufficiently similar efficiencies for noiseinduced transitions to occur between them. Interestingly, the authors already appear to have encountered signs of limitations when none of the effects in the cavity is dominant. Looking beyond laser physics and considering the remarkable success of the lowest-loss dictum, any significant progress towards understanding it more deeply may be relevant to a large class of self-organizing systems that have no evident connection to lasers.

The advance made by Wright and co-workers gives us reason to be optimistic that it is possible to develop a general theory of mode-locking. Presently, there are various theoretical formulations for regular mode-locking, and now a preliminary formulation for spatiotemporal modelocking. Each of these predicts the overall characteristics of the mode-locked state for a given cavity layout - within certain limitations. However, this is a limited level of understanding for a self-organized system where the final state is an emergent one. None can dissect the requirements to a new mode-locking state with desired features and translate them to a sequence of cavity elements with appropriate parameters. Consequently, laser design involves laboriously searching the vast parameter space or trying to adopt a previously known mode-locking state. A satisfactory solution to this inverse problem could revolutionize the development of mode-locked lasers.

The recent proposal of a thermodynamic treatment of nonlinear propagation of light in optical fibres with a large number of transverse modes ${ }^{4}$ may hold clues to a radically different approach. Unfortunately, this thermodynamic formulation is restricted to light propagation without loss or gain, without which mode-locking is impossible. But a generalization of the attractor dissection method guided by thermodynamics may be able to solve the inverse problem of mode-locking.

\section{F. Ömer Ilday \\ Department of Physics, Department of Electrical and Electronics Engineering, UNAM - National Nanotechnology Research Center, Bilkent University, Ankara, Turkey. \\ e-mail: ilday@bilkent.edu.tr}

Published online: 10 February 2020

https://doi.org/10.1038/s41567-020-0811-2

References

1. Wright, L. G. et al. Nat. Phys. https://doi.org/10.1038/s41567-020 0784-1 (2020).

2. Wright, L. G., Christodoulides, D. N. \& Wise, F. W. Science 358 , 94-97 (2017).

3. Haken, H. Light: Laser Light Dynamics (North-Holland, 1985).

4. Wu, F. O., Hassan, A. U. \& Christodoulides, D. N. Nat. Photon. 13, 776-782 (2019). 